\title{
Prognostic and diagnostic significance of Cavin 2 in lung adenocarcinoma
}

Xianguo Wang ${ }^{1}$, Diana Maria Cespedes Arcani ${ }^{1}$, Jingping Zhao ${ }^{1}$, Ming X ${ }^{1}$, Xuefeng Zhou ${ }^{1}$, Yibin Yang ${ }^{2}$

\author{
${ }^{1}$ Department of Thoracic and Cardiovascular Surgery, Zhongnan Hospital \\ of Wuhan University, Wuhan City, Hubei, China \\ 2Department of Respiratory Medicine, Zhongnan Hospital of Wuhan University, \\ Wuhan City, Hubei, China
}

Submitted: 4 November 2018

Accepted: 12 February 2019

Arch Med Sci 2020; 16 (5): 1189-1195

DOI: https://doi.org/10.5114/aoms.2019.85347

Copyright $\odot 2019$ Termedia \& Banach

\author{
Corresponding authors: \\ Xuefeng Zhou and Yibin Yang \\ 169 Donghu Road \\ Wuchang District \\ Wuhan city 430000 \\ Hubei, China \\ E-mail: zhou20180721@163. \\ com, zhou20180721@163. \\ com (Xuefeng Zhou) \\ 1594559173@qq.com \\ (Yibin Yang)
}

\begin{abstract}
Introduction: Cavin 2 down-regulation is reported in several malignant tumors and is associated with tumor progression. However, the role of Cavin 2 in lung adenocarcinoma is unknown. This study aimed to investigate the prognostic and diagnostic significance of Cavin 2 in lung adenocarcinoma. Material and methods: Cavin 2 expression levels were examined in 150 cases of lung adenocarcinoma and matched adjacent normal lung tissues using RNA extraction and reverse transcription-quantitative polymerase chain reaction (RT-qPCR), Western blotting and immunohistochemistry (IHC) assays. Then, the relationship of Cavin 2 expression with clinicopathological characteristics and patients' survival was further evaluated in lung adenocarcinoma.

Results: QPCR and Western blotting analysis indicated that Cavin 2 expression levels were significantly lower in lung adenocarcinoma tissues compared with those in adjacent normal lung tissues $(p<0.0001)$. The IHC results showed that positive expression of Cavin 2 was mainly located in cytoplasm as brown, but was hard to detect in lung adenocarcinoma tissues. The low-expression rates of Cavin 2 in lung adenocarcinoma and adjacent normal lung tissues were $62.0 \%$ and $20.0 \%$, respectively, and the difference was significant $(p<0.0001)$. Lower expression of Cavin 2 was significantly associated with tumor size, TNM stage and lymph node metastasis $(p<0.05)$. Conclusions: Cavin 2 has low expression in lung adenocarcinoma, which might be regarded as a potential prognostic and diagnostic biomarker.
\end{abstract}

Key words: Cavin 2, prognosis, lung cancer.

\section{Introduction}

Adenocarcinoma is the most common subtype of non-small cell lung cancer, which comprises more than $40 \%$ of lung cancers [1-3]. Surgical resection is the main treatment method for adenocarcinoma patients; however, patients' survival is still poor [4-6]. The overall survival rate of patients with lung adenocarcinoma is $13-58.3 \%$ [7]. The main reason is that lung adenocarcinoma tends to develop distant metastases at the early stage [8-11]. Identifying the potential molecular biomarkers in lung adenocarcinoma is essential for patients to select the best treatment strategy and improve outcomes.

Cavin 2, a membrane-bound phosphatidylserine-binding protein, is regarded as a critical organizer of caveolae and a regulator of angiogenesis $[12,13]$. Cavin 2 is required for angiogenesis and plays a key 
role in cell proliferation, migration and invasion $[14,15]$. Cavin 2 is expressed in heart and lung, but is hard to detect in pancreas, brain, kidney, skeletal muscle, liver and placenta [16]. A recent study showed that Cavin 2 is mostly expressed in the well-differentiated liposarcoma and correlates with the differentiation and proliferation of tumor cells [17]. Cavin 2 is significantly down-regulated in oral squamous cell carcinoma and associated with tumor progression [18]. However, the role of Cavin 2 in lung adenocarcinoma remains unclear.

In this study, we detected the expression levels of Cavin 2 in 150 cases of lung adenocarcinoma and matched adjacent normal lung tissues, and also evaluated the relationship of Cavin 2 with clinicopathological features and patients' survival.

\section{Material and methods}

\section{Patients and samples}

A total of 150 cases of lung adenocarcinoma and matched adjacent normal lung tissues were collected from Zhongnan Hospital of Wuhan University from 2009 to 2017 . The age of patients ranged from 28 to 76 years (mean: 50 years). None of the patients were treated with radiotherapy or chemotherapy before the surgical operation. Adjacent normal lung tissues were collected more than $3 \mathrm{~cm}$ far away from the tumor. Clinicopathological features such as age, sex, smoking history, tumor size, tumor differentiation, lymph node metastasis and TNM stage (UICC, 2009) were obtained from hospital records. Pathological diagnosis was performed by two pathologists in Zhongnan Hospital of Wuhan University. Follow-up time was recorded on the surgery day and lasted from 7 to 80 months (mean: 38.5 months). This study is supported by the Ethnics Committee of Zhongnan Hospital of Wuhan University (No. Z201603322).

\section{RNA extraction and reverse transcription- quantitative polymerase chain reaction (RT-qPCR)}

All tissues were collected and stored in liquid nitrogen. Total RNAs were isolated using RNAiso PLUS (Invitrogen, California, USA), and reverse transcribed into cDNA by the cDNA Synthesis Kit (TaKaRa Corp, Dalian, China). According to manufacturer's protocol, qPCR was performed using the 7500 SYBR Green Fast Real-Time PCR System ( $A B I$ Corp, USA). The reaction conditions were as follows: $95^{\circ} \mathrm{C}$ for $10 \mathrm{~min}, 95^{\circ} \mathrm{C}$ for $15 \mathrm{~s}$ in 40 cycles and $60^{\circ} \mathrm{C}$ for $60 \mathrm{~s}$. The primer sequences of Cavin 2 were 5'-TTGTGAAGGAGCCAGTTCCC-3' and 5'-TCAGAGGAGAGGTCCACGTT-3'. The primer sequences of GAPDH were 5'-CCCCGCTACTCCTCCTCCTAAG-3' and 5'-TCCACGACCAGTTGTCCATTCC-3'. Relative
mRNA expression was analyzed by the $2^{-\Delta \Delta c t}$ method $[19,20]$.

\section{Western blotting}

Using protease inhibitors, total proteins were extracted. $50 \mu \mathrm{g}$ of protein was detached by $10 \%$ SDS-PAGE and transferred to the polyvinylidene fluoride membranes. Membranes were blocked by $5 \%$ fat-free milk and incubated with Cavin 2 antibody ( 1 : 2500, Abcam Corp, USA) and GAPDH antibodies (1:2000, Zhongshan Corp, Beijing, China) overnight at $4^{\circ} \mathrm{C}$, respectively. Then, membranes were washed with PBST and incubated with a rabbit anti-goat horseradish peroxidase-conjugated secondary antibody for $30 \mathrm{~min}$ at room temperature. Protein blots were detected by enhanced chemiluminescence detector.

\section{Immunohistochemistry (IHC)}

Sections (2- $\mu$ m-thick) were obtained and deparaffinized with xylene and gradient ethanol. Antigen retrieval was performed with $3 \%$ hydrogen peroxide and $0.01 \mathrm{~mol} / \mathrm{l}$ sodium citrate buffer ( $\mathrm{pH}$ 6.0) at room temperature for $10 \mathrm{~min}$. Then, sections were washed with phosphate buffered saline (PBS) solution and incubated with Cavin 2 antibody (1 : 100, Abcam Corp, USA). Finally, sections were incubated with horseradish peroxidase-labeled goat anti-mouse/rabbit IgG polymer for $40 \mathrm{~min}$ at room temperature. Signals were detected by diaminobenzidine tetrahydrochloride (DAB).

Based on staining intensity and positive cell percentage, a semi-quantitated method was used to analyze IHC results. Staining intensity was scored as follows: blank (0), weak (1), moderate (2) and strong (3). Positive cell percentage was scored as follows: $<5 \%(0), \geq 5 \%$ to $<25 \%$ (1), $25 \%$ to $50 \%$ (2) and $>50 \%$ (3). Total score of each section ranged from 0 to 9 , which was computed by multiplying these two values. Based on ROC analysis, a score $\geq 4$ was regarded as high Cavin 2 expression and others were low Cavin 2 expression $(<4)$.

\section{Statistical analysis}

Data were recorded as mean \pm standard deviation (SD) and analyzed using SPSS software (version 19.0; SPSS, Chicago, IL, USA). As data did not have a normal distribution, the non-parametric Mann-Whitney $U$-test was used to compare the differences between two groups. The correlation between Cavin 2 expression and clinicopathological characters was analyzed by chi-square test $\left(\chi^{2}\right)$. Survival analysis was evaluated using the Kaplan-Meier method and Cox's proportional hazards model. $P<0.05$ was regarded as statistically significant. 


\section{Results}

Cavin 2 is lowly expressed in lung adenocarcinoma

As shown in Figure 1, the mRNA levels of Cavin 2 were significantly lower in lung adenocarcinoma tissues compared with those in matched adjacent normal lung tissues $(p<0.0001)$. Moreover, Western blot results showed that Cavin 2 protein levels were also lower in lung adenocarcinoma tissues compared with those in matched adjacent normal lung tissues (Figure 1 B). Subsequently, Cavin 2 expression was further detected by IHC (Fig- ures $1 \mathrm{C}-\mathrm{F})$. The results showed that positive expression of Cavin 2 was mainly located in cytoplasm as brown (Figures $1 \mathrm{D}$ and F). Based on ROC analysis of the $\mathrm{IHC}$ score, score $=4$ (sensitivity = 0.815 , specificity $=0.608$ ) was considered as the cut-off to differentiate lung adenoma and normal lung tissues (Figure 2). The low expression rates of Cavin 2 in lung adenocarcinoma and adjacent normal lung tissues were $62.0 \%$ and $20.0 \%$, respectively, and the difference was statistically significant $(p<0.0001$, Table I). These data indicated that Cavin 2 was lowly expressed in lung adenocarcinoma.
A
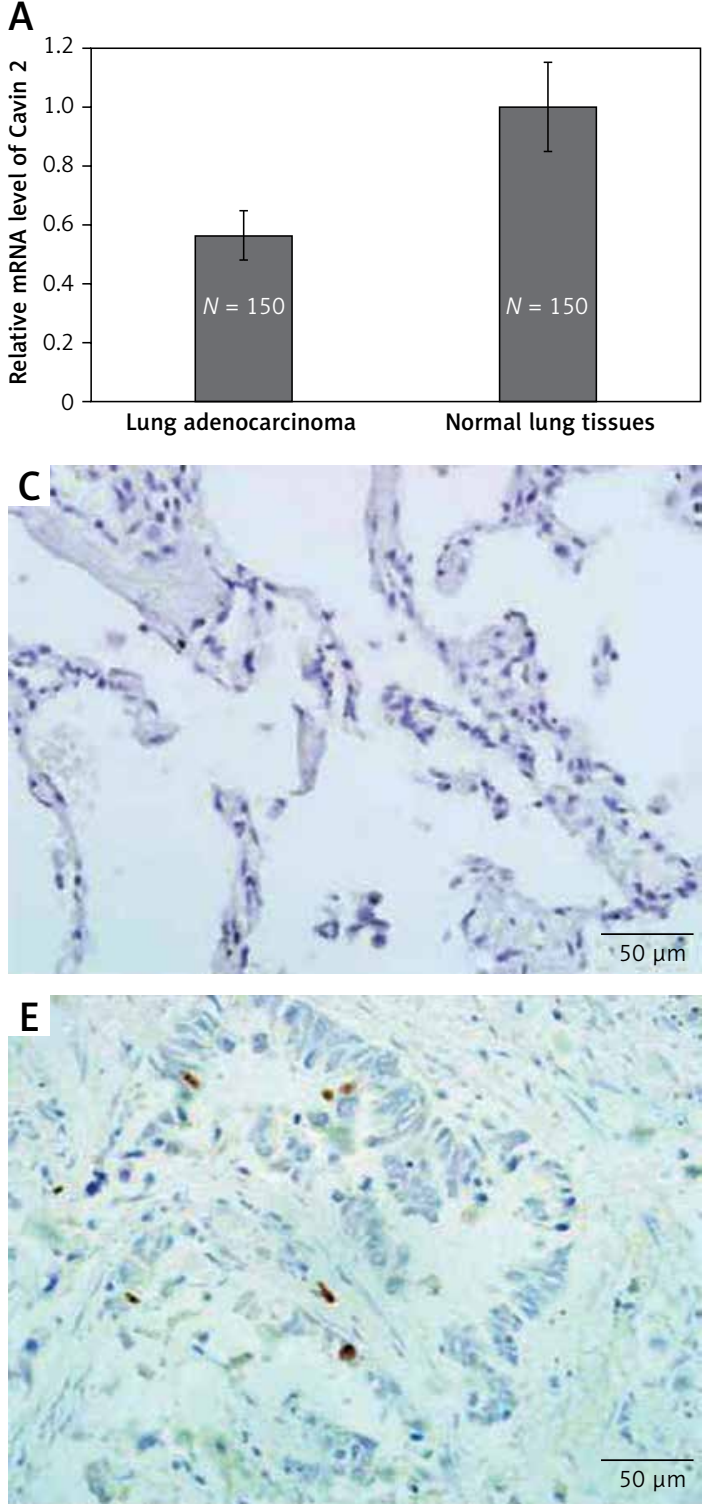

B

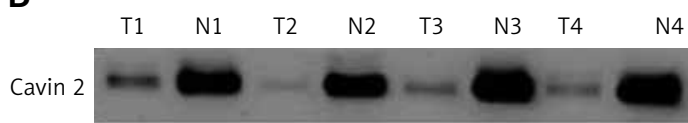

GAPDH

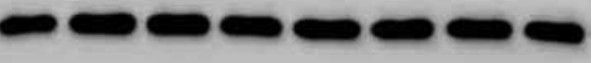

Figure 1. Cavin 2 was lowly expressed in lung adenocarcinoma. A - Cavin 2 mRNA level was measured in lung adenocarcinoma and normal lung tissues by RT-GPCR. B - Cavin 2 protein expression level was detected in lung adenocarcinoma and normal lung tissues by Western blotting. C - Low Cavin 2 expression was observed in normal lung tissues by IHC. D - High Cavin 2 expression was observed in normal lung tissues by IHC. E - Low Cavin 2 expression was observed in lung adenocarcinoma tissues by IHC. F - High Cavin 2 expression was observed in lung adenocarcinoma tissues by IHC

${ }^{*} P<0.0001, N$ - normal lung tissue, $T$ - tumor tissue (lung adenocarcinoma). 


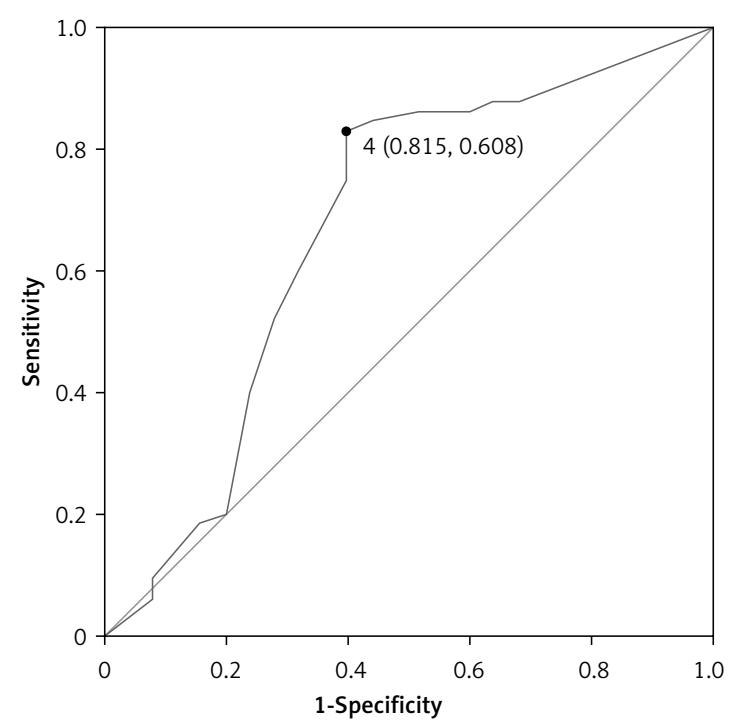

Figure 2. Score = 4 was selected as the cut-off by ROC analysis and the sensitivity and specificity were 0.815 and 0.608 , respectively

\section{Cavin 2 expression correlates with clinicopathological characters and predicts prognosis in lung adenocarcinoma}

Based on IHC analysis, the correlation between Cavin 2 expression and clinicopathological characters was analyzed in lung adenocarcinoma. The results showed that lower expression of Cavin 2 was significantly associated with tumor size, lymph node metastasis and TNM stage (Table II, $p<0.05)$, while it was not associated with age, sex, smoking history or tumor differentiation ( $\mathrm{Ta}$ ble II, $p>0.05$ ). Cavin 2 low expression was more easily observed in patients with lymph node metastasis. Patients with stage III presented lower levels of Cavin 2 compared with those with stage I-II. In addition, the correlation between Cavin 2 mRNA expression level and TNM stage was analyzed. As shown in Figure 3, the mRNA level of Cavin 2 in patients with stage III was significantly lower compared with those with stage I-II $(p<0.0001)$.

Then, the association between Cavin 2 expression and prognosis was analyzed by Kaplan-Meier method and Cox's proportional hazards model. Kaplan-Meier survival analysis showed that the median survival time of patients with low Cavin 2 expression was 45 months, which was significant- ly shorter than that of patients with high Cavin 2 expression (66 months) (Table III, Figure 4, $p<$ $0.0001)$. In addition, patients' prognosis was associated with lymph node metastasis and TNM stage (Table III, $p<0.05$ ), while it was not connected with age, sex, smoking history, tumor size or tumor differentiation (Table III, $p>0.05$ ). Cox regression survival analysis showed that Cavin 2 , lymph node metastasis and TNM stage were independent factors for predicting patients' prognosis in lung adenocarcinoma (Table IV, $p<0.05$ ).

\section{Discussion}

Cavin 2, a serum deprivation protein response gene, is enriched in lung tissue [12]. In this study, our data showed that low Cavin 2 expression was more easily observed in lung adenocarcinoma compared with normal lung tissues, indicating that Cavin 2 might be a tumor suppressor gene and Cavin 2 depletion might be implicated in the formation of lung adenocarcinoma. Moreover, Cavin 2 was also lowly expressed in oral cancer, prostate cancer, kidney cancer, breast cancers and hepatocellular carcinoma, which was consistent with our findings [21-25]. However, Codenotti et al. reported that Cavin 2, along with Cavin 1, was mostly expressed in the well-differentiated liposarcoma, while being almost undetectable in the more aggressive tumors [17]. These data suggested that Cavin 2 expression might be connected with tumor heterogeneity.

In addition, the correlation between Cavin 2 expression and clinicopathological characters was evaluated in lung adenocarcinoma. The results showed that Cavin 2 expression was significantly associated with tumor size, suggesting that Cavin 2 depletion might contribute to cell growth and proliferation. Furthermore, Cavin 2 expression was confirmed to be correlated with lymph node metastasis and TNM stage, indicating that low Cavin 2 expression correlated with the progression of lung adenocarcinoma. Survival analysis indicated that Cavin 2 as well as lymph node metastasis and TNM stage correlated with patients' prognosis and could be independent prognostic factors in lung adenocarcinoma. Thus, these data indicated that Cavin 2 was a prognostic biomarker in lung adenocarcinoma. Cavin 2 depletion might promote tumor progression and result in an unfavorable

Table I. Cavin 2 expression was detected in lung adenocarcinoma by IHC

\begin{tabular}{|lccc|}
\hline Tissue types & $N$ & \multicolumn{3}{c|}{ Cavin 2 } & -value \\
\cline { 3 - 4 } & & High expression, $n(\%)$ & Low expression, $n(\%)$ \\
\hline Lung adenocarcinoma & 150 & $57(38.0)$ & $93(62.0)$ \\
\hline Normal lung & 150 & $120(80.0)$ & $30(20.0)$ \\
\hline
\end{tabular}


Table II. Cavin 2 expression correlated with clinicopathological characters in lung adenocarcinoma

\begin{tabular}{|c|c|c|c|c|}
\hline \multirow[t]{2}{*}{ Clinicopathological characters } & \multirow[t]{2}{*}{$N$} & \multicolumn{2}{|c|}{ Cavin 2} & \multirow[t]{2}{*}{$P$-value } \\
\hline & & High expression & Low expression & \\
\hline \multicolumn{5}{|l|}{ Age [years]: } \\
\hline$\leq 50$ & 85 & 29 & 56 & 0.309 \\
\hline$>50$ & 65 & 28 & 37 & \\
\hline \multicolumn{5}{|l|}{ Sex: } \\
\hline Male & 84 & 28 & 56 & 0.236 \\
\hline Female & 66 & 29 & 37 & \\
\hline \multicolumn{5}{|l|}{ Smoking history: } \\
\hline Negative & 87 & 32 & 55 & 0.736 \\
\hline Positive & 63 & 25 & 38 & \\
\hline \multicolumn{5}{|l|}{ Tumor size $[\mathrm{cm}]$ : } \\
\hline$\leq 3$ & 42 & 22 & 20 & 0.026 \\
\hline$>3$ & 108 & 35 & 73 & \\
\hline \multicolumn{5}{|l|}{ Tumor differentiation: } \\
\hline High-middle grade & 107 & 39 & 68 & 0.579 \\
\hline Low grade & 43 & 18 & 25 & \\
\hline \multicolumn{5}{|l|}{ Lymph node metastasis: } \\
\hline Negative & 81 & 42 & 39 & $<0.0001$ \\
\hline Positive & 69 & 15 & 54 & \\
\hline \multicolumn{5}{|l|}{ TNM stage: } \\
\hline$|-| \mid$ & 87 & 44 & 43 & $<0.0001$ \\
\hline III & 63 & 13 & 50 & \\
\hline
\end{tabular}

prognosis. Unozawa et al. reported that Cavin 2 was a potential predictor for tumor progression in oral cancer [18]. In hepatocellular carcinoma, Cavin 2 was regarded as a potential diagnostic and prognostic biomarker [24].

Recently, Cavin 2 was reported to be involved in cell differentiation, proliferation, migration and invasion. Codenotti et al. reported that Cavin 2 was connected with adipogenic differentiation [17]. Unozawa et al. reported that Cavin 2 up-regulation slowed cellular proliferation by regulating cell-cycle arrest in oral cancer [18]. Boopathy et al. reported that Cavin 2 was required for endothelial cell proliferation, invasion and migration [12]. However, the effect of Cavin 2 expression on cell differentiation, proliferation, migration and invasion is still unclear in lung adenocarcinoma. Cavin 2 depletion may promote the progression of lung adenocarcinoma by regulating cell differentiation, proliferation, migration or invasion. However, this hypothesis needs to be validated by more evidence in future.

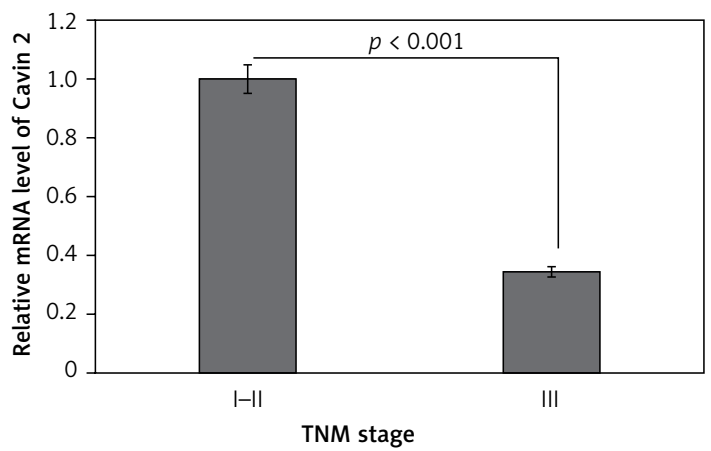

Figure 3. Lower mRNA level of Cavin 2 correlated with TNM stage

In conclusion, these data indicate that Cavin 2 is lowly expressed in lung adenocarcinoma and correlates with tumor formation and progression, which may be regarded as a potential prognostic and diagnostic biomarker in lung adenocarcinoma. Cavin 2 depletion forebodes poorer prognosis, which may be helpful for prognosis assessment in lung adenocarcinoma. However, there are some 
Table III. Survival analysis was performed by Kaplan-Meier method

\begin{tabular}{|c|c|c|c|}
\hline Variables & $N$ & Survival time, median $(95 \% \mathrm{Cl})$ [months] & $P$-value \\
\hline \multicolumn{4}{|l|}{ Cavin 2 expression: } \\
\hline High expression & 57 & $66(61-70)$ & $<0.0001$ \\
\hline Low expression & 93 & $45(42-48)$ & \\
\hline \multicolumn{4}{|l|}{ Age [years]: } \\
\hline$\leq 50$ & 85 & $59(52-66)$ & 0.259 \\
\hline$>50$ & 65 & $53(49-56)$ & \\
\hline \multicolumn{4}{|l|}{ Sex: } \\
\hline Male & 84 & $52(49-57)$ & 0.489 \\
\hline Female & 66 & $57(51-63)$ & \\
\hline \multicolumn{4}{|l|}{ Smoking history: } \\
\hline Negative & 87 & $54(49-59)$ & 0.558 \\
\hline Positive & 63 & $55(50-59)$ & \\
\hline \multicolumn{4}{|l|}{ Tumor size $[\mathrm{cm}]$ : } \\
\hline$\leq 3$ & 42 & $61(53-68)$ & 0.186 \\
\hline$>3$ & 108 & $53(49-56)$ & \\
\hline \multicolumn{4}{|l|}{ Tumor differentiation: } \\
\hline High-middle grade & 107 & $57(53-61)$ & 0.426 \\
\hline Low grade & 43 & $48(44-52)$ & \\
\hline \multicolumn{4}{|c|}{ Lymph node metastasis: } \\
\hline Negative & 81 & $62(57-67)$ & 0.001 \\
\hline Positive & 69 & $48(44-51)$ & \\
\hline \multicolumn{4}{|l|}{ TNM stage: } \\
\hline$|-| \mid$ & 87 & $63(58-68)$ & $<0.0001$ \\
\hline III & 63 & $44(40-47)$ & \\
\hline
\end{tabular}

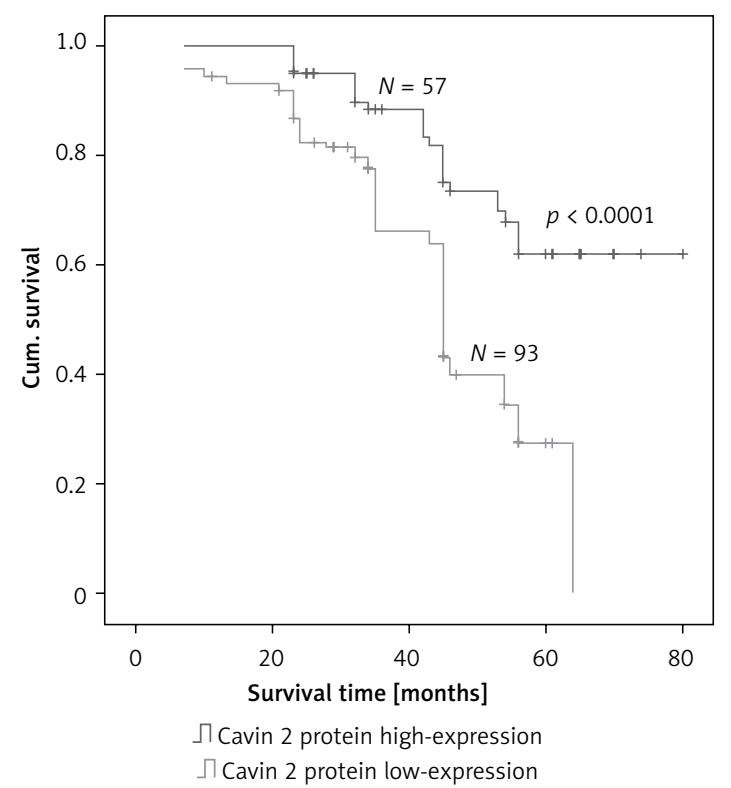

Figure 4. Kaplan-Meier analysis showed that the protein level of Cavin 2 correlated with prognosis in lung adenocarcinoma limitations of the current study. For one thing, all patients were from a single clinical center, which means it is hard to eliminate the selection bias. For another, the biological functions and mechanism of Cavin 2 in lung adenocarcinoma remain unknown. Therefore, further investigations are needed.

\section{Acknowledgments}

Xianguo Wang and Diana Maria Cespedes Arcani are the co-first authors.

\section{Conflict of interest}

The authors declare no conflict of interest. 
Table IV. Prognostic factors were further evaluated by multivariate Cox regression

\begin{tabular}{|lccc|}
\hline Variables & Hazard rate & $95 \% \mathrm{Cl}$ & $P$-value \\
\hline Cavin 2 (low expression vs. high expression ) & 2.716 & $1.681-4.387$ & $<0.0001$ \\
\hline Lymph node metastasis (positive vs. negative) & 2.088 & $1.332-3.270$ & 0.001 \\
\hline TNM stage (III vs. I-II) & 2.492 & $1.615-3.847$ & $<0.0001$ \\
\hline Age (> 50 years vs. $\leq 50)$ & 1.354 & $0.642-2.857$ & 0.426 \\
\hline Sex (male vs. female) & 0.858 & $0.360-2.041$ & 0.728 \\
\hline Smoking history (positive vs. negative) & 0.735 & $0.440-1.227$ & 0.239 \\
\hline Tumor size (> 3 cm vs. $\leq 3$ cm) & 1.485 & $0.700-3.15$ & 0.302 \\
\hline Tumor differentiation (low grade vs. $\leq$ high-middle grade) & 1.093 & $0.654-1.828$ & 0.734 \\
\hline
\end{tabular}

\section{References}

1. Lei B, Qi W, Zhao Y, et al. PBK/TOPK expression correlates with mutant $\mathrm{p} 53$ and affects patients' prognosis and cell proliferation and viability in lung adenocarcinoma. Hum Pathol 2015; 46: 217-24.

2. Liu H, Qie P, Yang G, et al. miR-181b inhibits chemoresistance in cisplatin-resistant $\mathrm{H} 446$ small cell lung cancer cells by targeting Bcl-2. Arch Med Sci 2018; 14: 745-51.

3. Lortet-Tieulent J, Soerjomataram I, Ferlay J, et al. International trends in lung cancer incidence by histological subtype: adenocarcinoma stabilizing in men but still increasing in women. Lung Cancer 2014; 84: 13-22.

4. Liu S, Li Y, Qi W, et al. Expression of Tiam1 predicts lymph node metastasis and poor survival of lung adenocarcinoma patients. Diagn Pathol 2014; 9: 69.

5. Chen X, Fu Y, Xu H, et al. SOX5 predicts poor prognosis in lung adenocarcinoma and promotes tumor metastasis through epithelial-mesenchymal transition. Oncotarget 2018; 9: 10891-4.

6. Li C, Kuo SW, Hsu HH, et al. Lung adenocarcinoma with intraoperatively diagnosed pleural seeding: Is main tumor resection beneficial for prognosis? J Thorac Cardiovasc Surg 2018; 155: 1238-49e1231.

7. Zhao S, Shen W, Yu J, et al. TBX21 predicts prognosis of patients and drives cancer stem cell maintenance via the TBX21-IL-4 pathway in lung adenocarcinoma. Stem Cell Res Ther 2018; 9: 89.

8. Hung JJ. Histologic subtype component predicts lymph node micrometastasis and prognosis in patients with stage I lung adenocarcinoma. J Thorac Dis 2017; 9: 3623-5.

9. Bucciarelli PR, Tan KS, Chudgar NP, et al. BRMS1 expression in surgically resected lung adenocarcinoma predicts future metastases and is associated with a poor prognosis. J Thorac Oncol 2018; 13: 73-84.

10. Ettinger DS. Ten years of progress in non-small cell lung cancer. J Natl Compr Canc Netw 2012; 10: 292-5.

11. Sun Y, Chen Y, Li S, et al. NanoVelcro-captured CTC number concomitant with enhanced serum levels of MMP7 and MMP9 enables accurate prediction of metastasis and poor prognosis in patients with lung adenocarcinoma. Int J Nanomed 2017; 12: 6399-412.

12. Boopathy GTK, Kulkarni M, Ho SY, et al. Cavin 2 regulates the activity and stability of endothelial nitric-oxide synthase (eNOS) in angiogenesis. J Biol Chem 2017; 292: 17760-6.

13. Toufaily C, Charfi C, Annabi B, et al. A role for the cavin-3/matrix metalloproteinase- 9 signaling axis in the regulation of PMA-activated human HT1080 fibrosarco- ma cell neoplastic phenotype. Cancer Growth Metastasis 2014; 7: 43-51.

14. Yi JS, Mun DG, Lee $H$, et al. PTRF/cavin-1 is essential for multidrug resistance in cancer cells. J Proteome Res 2013; 12: 605-14.

15. Gupta R, Toufaily C, Annabi B. Caveolin and cavin family members: dual roles in cancer. Biochimie 2014; 107 : 188-202.

16. Gustincich S, Vatta P, Goruppi S, et al. The human serum deprivation response gene (SDPR) maps to 2q32-q33 and codes for a phosphatidylserine-binding protein. Genomics 1999; 57: 120-9.

17. Codenotti S, Vezzoli M, Poliani PL, et al. Cavin-2 is a specific marker for detection of well-differentiated liposarcoma. Biochem Biophys Res Commun 2017; 493: 660-5.

18. Unozawa M, Kasamatsu A, Higo M, et al. Cavin-2 in oral cancer: a potential predictor for tumor progression. Mol Carcinog 2016; 55: 1037-47.

19. Jędroszka D, Orzechowska M, Bednarek AK. Predictive values of Notch signalling in renal carcinoma. Arch Med Sci 2017; 13: 1249-54.

20. Jen J, Tang YA, Lu YH, et al. Oct4 transcriptionally regulates the expression of long non-coding RNAs NEAT1 and MALAT1 to promote lung cancer progression. Mol Cancer 2017; 16: 104.

21. Altintas DM, Allioli N, Decaussin M, et al. Differentially expressed androgen-regulated genes in androgen-sensitive tissues reveal potential biomarkers of early prostate cancer. PLoS One 2013; 8: e66278.

22. Ozturk S, Papageorgis P, Wong CK, et al. SDPR functions as a metastasis suppressor in breast cancer by promoting apoptosis. Proc Natl Acad Sci USA 2016; 113: 638-43.

23. Li X, Jia Z, Shen Y, et al. Coordinate suppression of sdpr and fhl 1 expression in tumors of the breast, kidney, and prostate. Cancer Sci 2008; 99: 1326-33.

24. Jing $W$, Luo P, Zhu M, et al. Prognostic and diagnostic significance of SDPR-Cavin-2 in hepatocellular carcinoma. Cell Physiol Biochem 2016; 39: 950-60.

25. Aung CS, Hill MM, Bastiani M, et al. PTRF-cavin-1 expression decreases the migration of PC3 prostate cancer cells: role of matrix metalloprotease 9. Eur J Cell Biol 2011; 90: 136-42. 